\title{
The 12th international conference on differentiation therapy: targeting the aberrant growth, differentiation and cell death programs of cancer cells
}

\author{
F Pettersson ${ }^{1}$, WH Miller Jr ${ }^{*, 1}$, C Nervi ${ }^{2}$, HJ Gronemeyer ${ }^{3}$, J Licht ${ }^{4}$, MS Tallman ${ }^{4}$, S Waxman $^{5}$ \\ Cell Death and Differentiation (2011) 18, 1231-1233; doi:10.1038/cdd.2010.173; published online 7 January 2011
}

The 12th International Conference on Differentiation Therapy, 11-14 November 2009, Chicago, IL, USA

The 12th International Conference on Differentiation therapy brought together clinicians and researchers to exchange scientific data and discuss therapeutic strategies for the induction of differentiation and apoptosis of cancer cells.

\section{Differentiation Therapy \\ Differentiation therapy was defined in leukemia cell lines as a therapy that induces cell-cycle arrest and commitment to a differentiation program, followed by terminal cell division and apoptosis. Differentiation is more difficult to define in solid tumors, but a general concept of specific targeting of an aberrant event can be utilized, as demonstrated by the abundance of new 'targeted agents' in clinical development. Sam Waxman (New York, USA) described how targeted disruption of the transcriptional repressor Sin3 reverted silencing of genes involved in cell growth and differentiation, leading to impaired growth and invasion of breast cancer cells in 3D cultures and in vivo.}

Acute promyelocytic leukemia. The only dramatically successful differentiation therapy in the clinic remains treatment of acute promyelocytic leukemia (APL) with alltrans-retinoic acid (ATRA) and arsenic trioxide (ATO). Induction of granulocytic differentiation by ATRA and ATO is linked to derepression of retinoic acid receptor (RAR) signaling through degradation of the oncoprotein PML-RAR $\alpha$. Strategies to improve and extend the activity of ATO beyond APL were discussed by Wilson $H$ Miller, Jr (Montreal, Quebec, Canada). Combining ATO with the vitamin E derivative trolox enhances the toxicity of ATO in tumor cells while protecting normal cells, and a novel arsenical, darinaparsin, shows a potentially unique mechanism of action and enhanced activity compared with ATO. An interesting clinical perspective was presented by Vikram Mathews (Vellore,
India), who reported that single-agent ATO for APL is useful in areas where resources are scarce. Ongoing trials in India will determine whether single-agent ATO can reduce the risk of relapses.

\section{Epigenetics-Its Impact on Cancer Therapy}

Epigenetic means a change in gene expression not accompanied by an altered DNA sequence. Many cancers display epigenetic changes, which may promote tumor development. Epigenetic drugs aim to restore an overall 'normal' gene expression, although their mechanisms of action are not fully understood.

Inhibitors of DNA methylation. Many tumor suppressor genes are silenced by methylation. Peter Jones (Los Angeles, CA, USA) discussed how DNA methylation affects chromatin structure and how DNA methyltransferases (DNMTs) and polycomb group repressive complexes, which regulate histone methylation, cooperate to silence gene expression. Jean-Pierre Issa (Houston, TX, USA) described how clinical responses in patients with myeloid leukemia or myelodysplastic syndrome correlate with activation of specific genes or microRNAs, rather that demethylation, which may be transient. Strategies to improve the activity of DNMT inhibitors (DNMTi) will include combinations with other epigenetic modifiers, including histone deacetylase inhibitors and polycomb inhibitors.

Inhibitors of protein acetylation. HDACi cause hyperacetylation of histones and nonhistone proteins. These drugs block cell-cycle progression, induce differentiation and/or apoptosis through numerous mechanisms. Ricky Johnstone (East Melbourne, Australia) reported that vorinostat 'primes' BCL-2 overexpressing tumors to the proapoptotic effect

\footnotetext{
'Lady Davis Institute and Segal Cancer Center of the Jewish General Hospital, McGill University, Montreal, Canada; ${ }^{2}$ University of Rome La Sapienza, Rome, Italy; ${ }^{3}$ Institut de Génétique et de Biologie Moléculaire et Cellulaire, Illkirch, France; ${ }^{4}$ Robert H. Lurie Comprehensive Cancer Center, Northwestern University Feinberg School of Medicine, Chicago, IL, USA and ${ }^{5}$ Mount Sinai School of Medicine, New York, NY, USA

*Corresponding author: WH Miller Jr, Lady Davis Institute, 3755 Cote-Ste-Catherine Rd Montreal, Quebec H3T 1E2, Canada. Tel: +514 3408222 ext 4365 ;

Fax: +514 340 7576; E-mail: wmiller@jgh.mcgill.ca
} 
of $\mathrm{ABT}-737$, a small molecule inhibitor of $\mathrm{BCL}-2, \mathrm{BCL}-\mathrm{XL}$ and $B C L-w$. This requires upregulation of the proapoptotic $\mathrm{BMF}$, as deletion of BMF suppressed the apoptotic effects of the combination. James Bradner (Boston, MA, USA) described efforts to develop selective HDAC6 inhibitors, which increase acetylation of tubulin and HSP90, inducing degradation of its client proteins. This illustrates the fact that HDACs are functionally different, as discussed by Arthur Zelent (London, UK).

Histone methylation. Several histone methylases and demethylases are implicated in activation or repression of transcription and are aberrantly expressed in tumors. Like HDACs, most of these enzymes probably have nonhistone targets. For example, LSD1 demethylates p53 and represses its transcriptional and proapoptotic activities. The histone methyltransferase MMSET is frequently overexpressed in multiple myeloma, and work from the laboratory of Jonathan Licht (Chicago, IL, USA) indicates that MMSET functions as a transcriptional repressor in vivo. ChIP-chip analysis to identify promoters bound by MMSET identified three transcription factors involved in B-cell development: XBP1, IRF2 and BCL6. Inhibitors of histone methylases and demethylases, including EZH2 and LSD1, are under investigation as potential antitumor agents able to reverse aberrant gene repression.

MicroRNA. miRs may provide new epigenetic/transcriptional targets of differentiation therapy. Carlo $M$ Croce (Columbus, OH, USA) presented studies showing deregulation of many miRs in cancer and the potential consequences for cancer promotion. Targeting specific miRs can achieve potent antitumor effects. Clara Nervi (Rome, Italy) reported a link between miR-223 epigenetic/transcriptional deregulation and leukemogenesis. The miR-223 gene is epigenetically silenced by the leukemia fusion AML1/ETO oncoprotein. Increased miR-223 activity subsequent to AML1/ETO downregulation or miR-223 ectopic expression triggers granulocytic differentiation of myeloid leukemias.

\section{Selective Apoptosis Activators}

The BCL-2 family of proteins controls mitochondrial outer membrane permeabilization (MOMP), triggering caspase activation and apoptosis, following various stimuli. Douglas Green (Memphis, TN, USA) mentioned that cell death occurring subsequent to MOMP can be caspase independent, presenting a potential new target for therapy. Michael Andreeff (Houston, TX, USA) talked about the tumor microenvironment causing resistance in vivo to treatments that work well in vitro. In fact, stromal cells co-cultured with leukemic cells can 'mimic' mutations found in the malignant cells, and display increased activation of ERK, AKT, and so on. New agents, such as CXCR4 and VLA4 inhibitors, work by disrupting leukemia stem cell microenvironment interactions. Hinrich Gronemeyer (Illkirch, France) discussed a novel triple-active drug (UVI5008) acting as an inhibitor of HDACs, sirtuins and DNMTs. UVI5008 displays tumor selective activity through induction of TNF-related apoptosis inducing ligand (TRAIL) and induction of reactive oxygen species.

\section{Targeted Therapy for Cancer: Present and Future}

Targeting signal transduction pathways. Ruibao Ren (Waltham, MA, USA) discussed the oncogene RAS, which is mutated or activated downstream of tyrosine kinase receptors in a large percentage of cancers. Targeting palmitoylation, which is one of several posttranslational modifications essential for RAS function, may be an effective therapeutic option in leukemia. AEG-1 is a downstream target of H-RAS and a potential therapeutic strategy for malignant glioma, as described by Paul Fisher (Richmond, VA, USA). Knock down of AEG1 with siRNAs in murine models resulted in inhibition of cell viability, cell invasion and cloning efficiency. The p38-MAP kinase pathway is constitutively activated in high-risk MDS. Leonidas Platanias (Chicago, IL, USA) showed that p38 inhibitors enhance hematopoietic colony formation in bone marrow samples of these patients. Fabrizio Galimberti (Hanover, NH, USA) talked about how targeting the CDK2cyclin $E$ complex can inhibit growth of lung cancers and suggested that Seliciclib, an inhibitor of CDK2, CDK7 and CDK9, may have synergistic antineoplastic effects in lung cancer when combined with taxanes.

Targeting the proteasome. Multiple myeloma (MM) is one of the best genetically characterized malignancies and defining the pathogenesis of MM has allowed development of successful therapies. Aggressive MM have high levels of NFKB activity, which underlies the sensitivity of MM cells to proteasome and IKK $\beta$ inhibitors. Kenneth Anderson (Boston, MA, USA) and Robert Orslowski (Houston, TX, USA) discussed the potential of combining bortezomib with other targeted agents, including HSP27 antisense and inhibitors of p38, HSP90, AKT, IL-6 and HDACs, to overcome resistance or enhance cytotoxicity. There are also new proteasome inhibitors, such as CEP-18770, carfilzomib, NPI-0052 and PR-924, a selective inhibitor of immunoproteasome subunit LMP-7.

\section{Cancer Stem Cells: The Ultimate Target?}

Cancers arise from tissue stem cells and/or progenitors with dysregulated self-renewal pathways, a process regulated by intrinsic factors (e.g. Notch, Hedgehog and Wnt) and signals from the microenvironment. Max Wicha (Ann Arbor, MI, USA), presented evidence that mesenchymal stem cells may stimulate breast tumor growth and form 'cancer stem cell niches'. Benjamin Neel (Ontario, Canada) established a system to isolate, enrich and assay cancer-initiating cells from primary papillary serous ovarian cancer based on cell surface/ functional marker expression and high-throughput flow cytometry methods. William Matsui (Baltimore, MD, USA) described the existence of MM cancer stem cells, a rare cell population resembling normal memory $B$ cells, which are relatively resistant to a wide variety of standard anti-cancer agents, suggesting their role in mediating tumor regrowth and relapse. Craig T Jordan (Rochester, NY, USA) discussed the intrinsic heterogeneity and variability of leukemia stem cell markers and provided evidence for an antileukemia activity of the small molecule parthenolide. Parthenolide inhibits NF-kB 
and HSP-70, increases reactive oxygen species, and induces apoptosis of primary acute myeloid leukemia stem/progenitor cells without affecting normal hematopoietic cells.

\section{Conclusions and Recommendations for Future Research}

The 12th ICDT provided an overview of therapeutic agents in development and potential targets for future therapies. With a large number of novel agents with limited single-agent activity, researchers must make a concerted effort to determine rational combinations of drugs before starting clinical trials. Therefore, research must focus on determining the true mechanisms of action of new and existing therapies so that better compounds, as well as possible synergistic or additive combinations, can be identified. Further work must also focus on new types of treatment, such as drugs targeting miR or histone methylation, as well as finding ways to cheaply, effectively and quickly identify patients who are candidates for specific targeted therapies.

\section{Conflict of Interest}

The authors declare no conflict of interest.

Acknowledgements. We thank the organizers and participants for their contribution to this successful meeting. Financial support was obtained from Bristol-Myers Squibb, Celgene, Cephalon, Eisai, Lilly, Millennium, Novartis and the Samuel Waxman Cancer Research Foundation. 